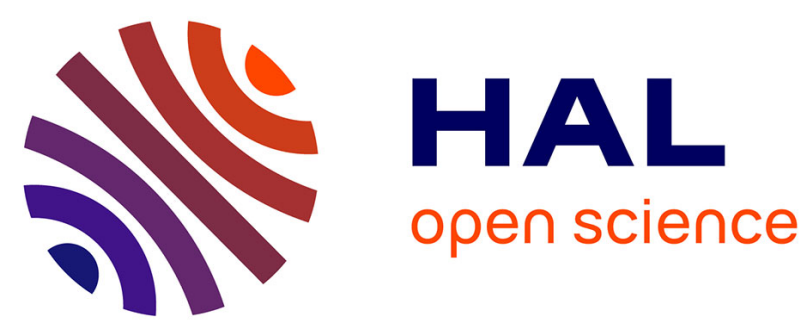

\title{
Image-Based Modeling of the Heterogeneity of Propagation of the Cardiac Action Potential. Example of Rat Heart High Resolution MRI
}

Anđela Davidović, Yves Coudière, Yves Bourgault

\section{- To cite this version:}

Anđela Davidović, Yves Coudière, Yves Bourgault. Image-Based Modeling of the Heterogeneity of Propagation of the Cardiac Action Potential. Example of Rat Heart High Resolution MRI. 9th international conference on Functional Imaging and Modeling of the Heart (FIMH 2017), Jun 2017, Toronto, Canada. pp.260-270, 10.1007/978-3-319-59448-4_25. hal-01563770

\author{
HAL Id: hal-01563770 \\ https://hal.science/hal-01563770
}

Submitted on 18 Jul 2017

HAL is a multi-disciplinary open access archive for the deposit and dissemination of scientific research documents, whether they are published or not. The documents may come from teaching and research institutions in France or abroad, or from public or private research centers.
L'archive ouverte pluridisciplinaire HAL, est destinée au dépôt et à la diffusion de documents scientifiques de niveau recherche, publiés ou non, émanant des établissements d'enseignement et de recherche français ou étrangers, des laboratoires publics ou privés. 


\title{
Image-based modeling of the heterogeneity of propagation of the cardiac action potential. Example of rat heart high resolution MRI.
}

\author{
Anđela Davidović ${ }^{1,2,3}$, Yves Coudière ${ }^{1,2,3,4}$, Yves Bourgault ${ }^{5}$ \\ ${ }^{1}$ Inria Bordeaux Sud-Ouest, Talence, France \\ ${ }^{2}$ IHU Liryc, Electrophysiology and Heart Modeling Institute, Pessac, France \\ ${ }^{3}$ Institute of Mathematics of Bordeaux, Talence, France \\ ${ }^{4}$ University of Bordeaux, Talence, France \\ ${ }^{5}$ University of Ottawa, Ontario, Canada
}

\begin{abstract}
In this paper we present a modified bidomain model, derived with homogenization technique from assumption of existence of diffusive inclusions in the cardiac tissue. The diffusive inclusions represent regions without electrically active myocytes, e.g. fat, fibrosis etc. We present the application of this model to a rat heart. Starting from high resolution (HR) MRI, geometry is built and meshed using image processing techniques. We perform a study on the effects of tissue heterogeneities induced with diffusive inclusions on the velocity and shape of the depolarization wavefront. We study several test cases with different geometries for diffusive inclusions, and we find that the velocity might be affected by $5 \%$ and up to $37 \%$ in some cases. Additionally, the shape of the wavefront is affected.
\end{abstract}

Keywords: bidomain model, heterogeneous conductivities, fibrosis, multiscale modelling, image-based modelling

\section{Introduction}

The standard macroscopic model for the electrophysiology of the heart is the bidomain model [10. This model is an anisotropic three-dimensional cable equation, that represents the averaged electric behavior of the myocardium. The electrical conductivity tensors for the intracellular and extracellular spaces are anisotropic, with the electrical conduction being the fastest in the fiber direction. In the standard bidomain model the anisotropy ratio in the intracellular space is about 10:1, while in the extracellular space about $2: 1$.

The bidomain model assumes the existence of uniformly spread myocytes, organized into a dense network [11, [14. It is a reasonable assumption for describing the propagation of the action potential in healthy tissues. In pathological cases this assumption does not hold, as there are regions with large patches of collagen or fibrosis. This is observed in ischemic and rheumatic heart disease, inflammation, hypertrophy, and infarction [3]. In the current modeling of such defects, usually the standard models for the healthy tissues are used, with the 
model parameters tuned in an ad hoc way.

An explanatory model was proposed for more rigorous tuning of the parameters in such situations, in [5] and [6]. The main extension of this model w.r.t. the standard bidomain model is the existence of relatively large regions of tissue where there are no myocytes, nor other kind of cells, and these regions are assumed to be passive electrical conductors. They are called diffusive inclusions. The diffusive inclusions may represent electrically passive infiltrations in the cardiac tissue like fat, collagen, fibrosis etc.

In this paper we apply the proposed model to a slab of rat heart. We start from HR-MRI data of a rat heart. We perform the image analysis to obtain a computational domain, to define the diffusive inclusions and to find the local volume fractions of the diffusive inclusions. These are then used in the simulations of the modified bidomain model. Finally, we make a study of how different diffusive inclusions might affect the shape and the velocity of depolarization waves.

\section{Modified bidomain model for rat heart}

In this section we give an overview of the model proposed in [5] and [6]. Full derivation of the model is out of the scope of this paper, since we want to focus on the application of this model on a real rat heart.

On the microscopic scale, periodic diffusive inclusions have been embedded into the healthy cardiac tissue, i.e. the bidomain model. In order to observe the effects of these inclusions on the macroscopic scale a homogenisation technique has been applied. This approach gives rise to the following two sets of problems:

- homogenised problem - that is in fact the macroscopic model that we were looking for. In our case it is a modified bidomain model, where the effects of inclusions are mainly contained in the conductivity tensors, as expected.

- cell problems - these are correction equations, used to calculate the modified conductivity tensors. They are set on the unit cube of $\mathbb{R}^{3}$ and represent a rescaled typical volume of interest of cardiac tissue.

The general homogenisation approach is presented in [1, 2]. It is a well known technique in mathematical modelling, and has been used for example in the derivation of the bidomain model [11, [14].

\section{$2.1 \quad$ Equations}

Homogenized problem. On the microscopic scale it is assumed that diffusive inclusions are periodic with a period $\epsilon$, such that $l \ll \epsilon \ll L$, where $L$ is the tissue scale and $l$ is the cardiac cell scale. Applying the mathematical homogenization technique, assuming that $\epsilon$ goes to zero, an averaged non-dimensional model has 
been derived, and is given as follows

$$
\begin{aligned}
\left(\partial_{t} v+I_{\text {ion }}(v, h)\right) \xi_{B} & =N \nabla \cdot\left(\sigma^{i *} \nabla u_{i}\right), & & \text { in }[0, T] \times \Omega, \\
\left(\partial_{t} v+I_{\text {ion }}(v, h)\right) \xi_{B} & =-N \nabla \cdot\left(\sigma^{e *} \nabla u_{e}\right), & & \text { in }[0, T] \times \Omega, \\
\partial_{t} h+g(v, h) & =0, & & \text { in }[0, T] \times \Omega,
\end{aligned}
$$

where $u_{i}$ and $u_{e}$ are the intracellular and extracellular potentials, $v=u_{i}-u_{e}$ is the transmembrane voltage, $I_{i o n}$ is the transmembrane current that is a function of $v$ and $h$, a state variable, $\xi_{B}$ is the local volume fraction of the healthy tissue, $N$ is the adimensionalisation parameter that depends on time and space scales and physical properties of the cell as given in [15], and $\sigma^{i *}$ and $\sigma^{e *}$ are the modified conductivity tensors.

Cell problems. The effective intracellular and extracellular conductivity tensors $\sigma_{i}^{*}$ and $\sigma_{e}^{*}$ are obtained by solving the set of so-called cell problems that are defined on the unit cell space, $Y=[0,1] \times[0,1] \times[0,1]$. The unit cell is in fact the rescaled periodic cell of tissue, where one can identify the healthy tissue, $Y_{B}$, and the diffusive inclusion, $Y_{D}$, see Figure 1. Note that $Y$ represents a microscopic piece of tissue (of size $\epsilon$ ), and the tissue is considered to be locally periodic. On a large scale the shape and size of the diffusive inclusions may vary, as illustrated in the following study.
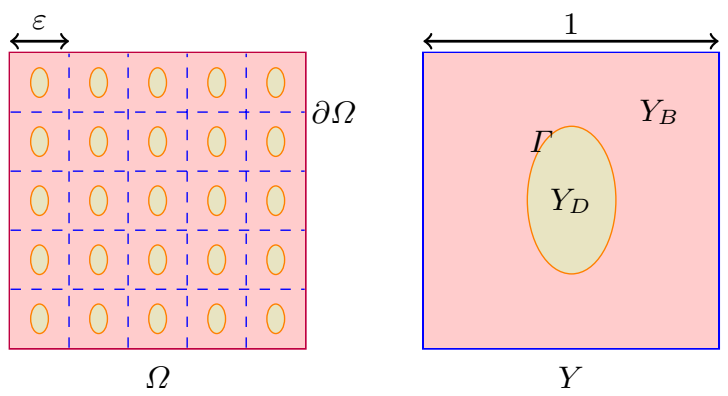

Fig. 1: Left: the idealised full domain, $\Omega$. Right: the unit cell, $Y$.

On the unit cell we define the $Y$-periodic functions $w_{j}$ and $w_{j}^{i}, j=1,2,3$, as solutions to the cell problems

$$
\begin{aligned}
& \nabla \cdot\left(\sigma_{i} \nabla w_{j}^{i}\right)=0, \text { in } Y_{B}, \quad \sigma_{i}\left(\nabla w_{j}^{i}+e_{j}\right) \cdot n=0, \text { on } \Gamma, \\
& \nabla \cdot\left(\sigma \nabla w_{j}\right)=0, \text { in } Y, \quad\left(\sigma_{e}-\sigma_{d}\right)\left(\nabla w_{j}+e_{j}\right) \cdot n=0, \text { on } \Gamma,
\end{aligned}
$$

where $\sigma_{i}$ and $\sigma_{e}$ are the intracellular and extracellular conductivity tensors used in the healthy tissue, i.e. in the standard bidomain model, and $\sigma_{d}$ is the conductivity assumed inside of the diffusive inclusion, $n$ is an outer normal to the 
boundary $\Gamma$ and $e_{j}, j=1,2,3$, are the unit vectors of the standard basis. The conductivity tensor $\sigma$ in (5) is defined as $\sigma=\sigma_{e}$, in $Y_{B}$, and $\sigma=\sigma_{d}$, in $Y_{D}$.

The periodic functions $w_{j}^{i}$ and $w_{j}$ are then used to define the effective conductivity tensors as:

$$
\begin{aligned}
\sigma_{k j}^{i *}= & \sigma_{i, k j} \xi_{B} \\
& +\left(\sigma_{i, k 1} \int_{Y_{B}} \partial_{y_{1}} w_{j}^{i} d y+\sigma_{i, k 2} \int_{Y_{B}} \partial_{y_{2}} w_{j}^{i} d y+\sigma_{i, k 3} \int_{Y_{B}} \partial_{y_{3}} w_{j}^{i} d y\right), \\
\sigma_{k j}^{e *}= & \int_{Y} \sigma_{k j} d y+\left(\int_{Y} \sigma_{k 1} \partial_{y_{1}} w_{j} d y+\int_{Y} \sigma_{k 2} \partial_{y_{2}} w_{j} d y+\int_{Y} \sigma_{k 3} \partial_{y_{3}} w_{j} d y\right),
\end{aligned}
$$

for $j, k=1,2,3$, and $\xi_{B}=\left|Y_{B}\right|$.

As one may notice, $\sigma^{i *}$ and $\sigma^{e *}$ depend not only on the volume fraction of the diffusive inclusion, but on their shape as well.

Ionic model for the rat heart. A reference ionic model that corresponds to the action potential (AP) of the rat heart is given in [13]. In our simulations we use the Mitchell Schaeffer (MS) model [9, fitted to the reference ionic model for the rat heart,

$$
\begin{aligned}
I_{i o n}(v, h) & =\frac{1}{\tau_{\text {in }}} h v^{2}(v-1)+\frac{1}{\tau_{\text {out }}} v, \\
g(v, h) & =\left\{\begin{array}{cl}
\frac{1}{\tau_{\text {open }}}(1-v), & \text { for } v<v_{\text {gate }} \\
-\frac{1}{\tau_{\text {close }}} v, & \text { for } v \geq v_{\text {gate }},
\end{array}\right.
\end{aligned}
$$

where $I_{i o n}, g, v$ and $h$ have the same meaning as before, and $\tau_{\text {in }}, \tau_{\text {out }}, \tau_{\text {open }}, \tau_{c l o s e}$, and $v_{\text {gate }}$ are the model parameters. The former four are normally given in units of time and are related to the duration of AP phases: depolarisation, repolarisation, plateau phase and the total AP duration. The parameter $v_{\text {gate }}$ is related to the threshold value of transmembrane voltage when the AP in cardiac cell is triggered.

The original MS model is given so that the transmembrane voltage, $v$, is non-dimensional and is scaled to $[0,1]$. Here we went a step further and used the approach given in [15] to work with a fully non-dimensional model, using the time scale $T=10^{-3} \mathrm{~s}$ and the length scale $L=10^{-3} \mathrm{~m}$. We have used the algorithm given in [12], that enables us to automatically determine new parameters of the MS model, given in Table 1 . Note that in the non-dimensional model the conductivities are also scaled with the parameter $\bar{\sigma}=0.1 \mathrm{Sm}^{-1}$, and the non-dimensional values for the anisotropic tensors $\sigma_{i}$ and $\sigma_{e}$ are given in Table 1 .

The MS model is chosen for its mathematical simplicity and easy numerical implementation. It has been shown that it is convenient as well for patientspecific modeling in [16]. 
Table 1: Non-dimensional bidomain model parameters of the rat heart, as in [15].

\begin{tabular}{|c|c|c|c|c|c|c|c|c|c|}
\hline$\tau_{\text {in }}$ & $\tau_{\text {out }}$ & $\tau_{\text {open }}$ & $\tau_{\text {close }}$ & $v_{\text {gate }}$ & $\sigma_{i, l}$ & $\sigma_{i, t}$ & $\sigma_{e, l}$ & $\sigma_{e, t}$ & $N$ \\
0.073 & 8.369 & 25.743 & 15.438 & 0.02 & 1.741 & 0.1934 & 3.906 & 1.97 & 0.0125 \\
\hline
\end{tabular}

\subsection{Computing effective conductivities}

To compute the effective conductivities $\sigma^{i *}$ and $\sigma^{e *}$, first we have to solve the equations (4)-(5) on the unit cell $Y$. Hence, we need values for the conductivities $\sigma_{i}, \sigma_{e}$ and $\sigma_{d}$. The values for the anisotropic tensors $\sigma_{i}$ and $\sigma_{e}$ corresponding to the non-dimensional model are given in Table 1 . We assume that the fibers are aligned with the x-axis, so $\sigma_{i}$ and $\sigma_{e}$ are diagonal matrices. In that sense, we have $\sigma_{i, 11}=\sigma_{i, l}$ and $\sigma_{i, 22}=\sigma_{i, 33}=\sigma_{i, t}$, with non-diagonal terms being equal to zero. Similarly, $\sigma_{e, 11}=\sigma_{e, l}$ and $\sigma_{e, 22}=\sigma_{e, 33}=\sigma_{e, t}$, with non-diagonal terms being equal to zero.

The conductivity tensor $\sigma_{d}$ is scaled with the same parameter $\bar{\sigma}=0.1 \mathrm{Sm}^{-1}$. We chose to test isotropic values $\sigma_{d}=0.2,1.5$ and 3.0, where the former one corresponds to the conductivity of fatty tissue and the latter to isotropic extracellular space. Several shapes of the diffusive inclusions have been tested, as shown in the Figure 2 .

The cell problems were solved using the finite element approach and FreeFem ++1 . To illustrate results we plot the conductivity tensors and the anisotropy ratios for one case (see Figure 3). In this case we obtain diagonal conductivity tensors with $\sigma_{22}^{i *}=\sigma_{33}^{i *}$ and $\sigma_{22}^{e *}=\sigma_{33}^{e *}$. We observe changes in the anisotropy ratios for both intra and extra-cellular conductivities. It is consistent with [6] where the shape of diffusive inclusions induces significant changes in anisotropy ratios.

Additionally to that, the main direction of propagation might be changed as well. This implies that in certain situations, depending on the tissue structure, one needs to know more than just the fiber direction to recover the correct wavefront of depolarization.

\section{Application to a slab of rat heart}

\subsection{Data on the rat heart}

HR-MRI. Data were provided by the IHU-Liryc, Bordeaux. The MR Imaging has been performed on the heart of a male Wistar rat. After the proper preparation of the heart, the heart was perfused with MRI contrast agent and fixative, and then stored in contrast/fixative solution until imaging. The heart was imaged using a T1 weighted FLASH (Fast Low Angle SHot) MRI sequence in a Bruker (Ettlingen, Germany) 9.4T spectroscope with 20 averages and echo time $(\mathrm{TE})=7.9 \mathrm{~ms}$, repetition time $(\mathrm{TR})=50 \mathrm{~ms}$, and flip angle 40 degrees, at a resolution of $50 \times 50 \times 50 \mu \mathrm{m}$, a matrix size of $256 \times 256 \times 512$ for a field of view of $12.8 \times 12.8 \times 25.6 \mathrm{~mm}$. For further details see $[8]$.

\footnotetext{
1 http://www.freefem.org/
} 

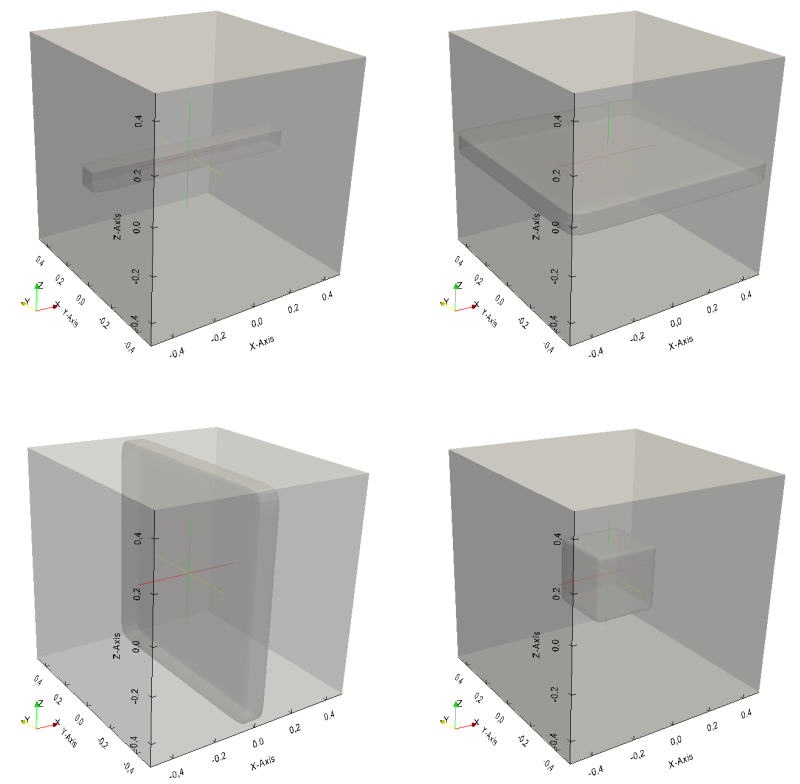

Fig. 2: Test cases for the unit cells in 3D. The shapes of the inclusions are: sticks aligned with fiber direction (here x-axis), plates parallel to fibers, plates perpendicular to fibers, and nearly cubic superellipsoids. For each case we used superellipsoids, with different semi-axes.
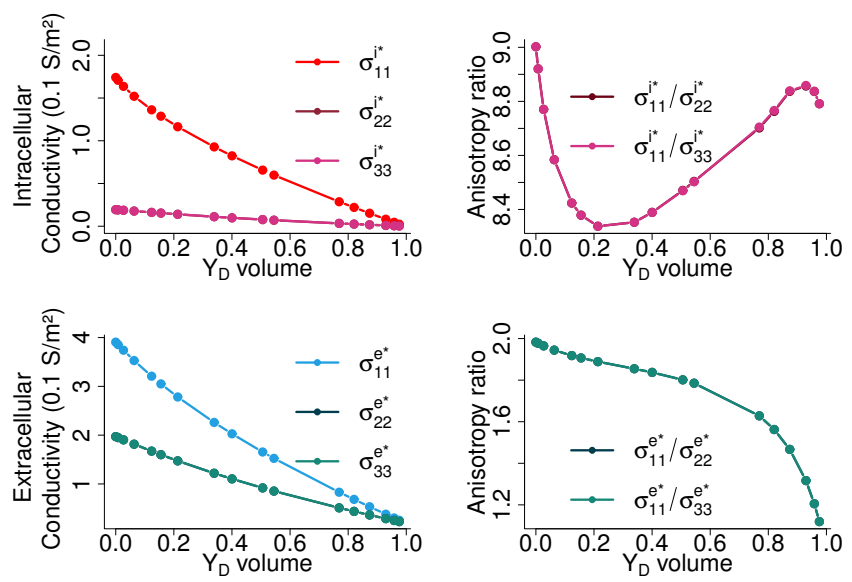

Fig. 3: Effective conductivities and anisotropy ratios for inclusions in the shape of cubes (see Figure 2 at the bottom right), ranging in volume fraction from 0 to $98 \%$, with fibers aligned with the $\mathrm{x}$-axis. 
DT-MRI fiber directions. The fiber structure in the cardiac tissue leads to anisotropy in the bidomain model. For the simulation of both bidomain and modified model it is important to assess the fiber orientation in the tissue. The diffusion tensor (DT) MRI technique is used for this purpose. The data from DT-MRI are given on an image that is four times coarser than the original HRMRI data, i.e. $64 \times 64 \times 128$ for the same view field. This results in a spacing between voxels that is four times larger, i.e. $0.2 \times 0.2 \times 0.2 \mathrm{~mm}$.

\subsection{Image processing}

Segmentation. The semi-manual segmentation of the images has been performed with the software $\operatorname{Seg} 3 D^{2}$. The median filter and thresholding on the gray scale were used to define roughly the boundaries of cardiac tissue. Following thresholding, bad pixels have been fixed manually, layer by layer. Cropping tools have been used to define the computational domain, i.e. a part of the leftventricular wall. This is done solely for performance reasons, in order to reduce the computing cost of the simulations.

Diffusive inclusion detection and local volume fraction. Using the software $S e g 3 D$ we were able to define the diffusive inclusions in the computational domain. For this we used only a threshold on gray scale, without additional processing, see Figure 4. The mask of computational domain with flagged subdomain of diffusive inclusions has been exported in .mat file, and Matlab was used for the computation of the local volume fraction of the inclusions. Around each voxel, $X$, we define a $5 \times 5 \times 5$ window, and count the number $n$ of voxels inside of this window that belong to the diffusive inclusions, $n \in[0,125]$. The local volume fraction of voxel $X$ is then given as $\xi_{D}(X)=\frac{n}{125}$.

Mesh generation. For the mesh generation we used the software SCIRun ${ }^{3}$ which integrates the call function to tetgen. We have set a minimum radius-edge ratio and imposed a maximum volume constraint on tetrahedra. The result is a fine mesh, that has 351706 nodes and 1924747 tetrahedra, see Figure 4.

Mapping data on the mesh. To be able to use the imaging data in our simulations we have to map them on the mesh nodes. For this purpose, we use the software SCIRun. Both, local volume fraction $\xi_{D}$ and the fiber orientations were mapped on nodes of the mesh.

2 http://www.sci.utah.edu/cibc-software/seg3d.html

3 http://www.sci.utah.edu/cibc-software/scirun.html 

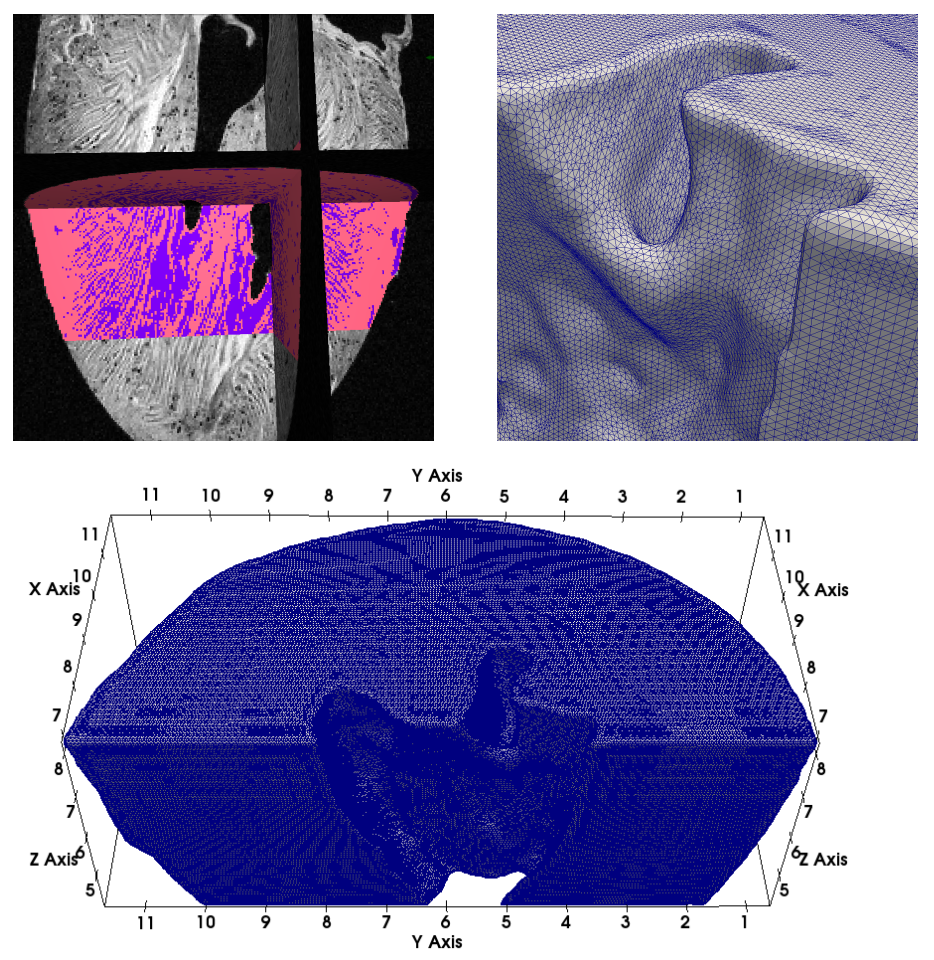

Fig. 4: Up left: segmentation from the HR-MRI data and diffusive inclusions detection. Bottom: fine mesh of the selected domain. Up right: details of the mesh.

\subsection{Simulations}

Settings. Here we set the parameters for the bidomain and modified model (1)-(3). The computational mesh and $\xi_{B}=1-\xi_{D}$ are set. The non-dimensional parameter $N$ is set to 0.0125 , as in the Table 1 . The effective conductivity tensors, $\sigma_{i}^{*}$ and $\sigma_{e}^{*}$, have been computed for several test cases, as in Figure 2 with the fiber direction assumed to be aligned with the $\mathrm{x}$-axis and for the range of diffusive inclusions volume fractions $\xi_{D} \in(0,1)$. Now, for each node in the mesh we assign one of these values depending on the corresponding local value of $\xi_{D}$, obtained from the image processing. Finally, we recompute the effective conductivities for each node based on the fiber direction, obtained from DT-MRI images.

We use linear finite elements as implemented in FreeFem ++ to solve the the bidomain and modified model (1)-(3). For the time discretization we use the semi-explicit SBDF2 numerical scheme, as proposed in [7, with the time step $d t=0.05 \mathrm{~ms}$. The resulting linear system was solved using the conjugate gradient method, to avoid excessive memory usage. 
Results. We run simulations for several test cases as given in Table 2, The reference case consists in solving the standard bidomain model without any diffusive inclusions. In the table we report the total depolarization duration of the computational domain, $T_{D}$, calculated as the first time $t>0$ for which all nodes have a value $v>v_{\text {gate }}$. From this one we conclude that the velocity is affected by $5-7 \%$ in most of the cases, and $35 \%$ and $37 \%$ in the case of plates perpendicular to the fiber direction.

On Figure 5 we plot the isochrones of depolarization on the boundary of the computational domain. We compare the reference case to the case of inclusions with the shapes of plates parallel to the fiber directions. We can observe a change in the shape of the wavefront. The same isochrones plotted on a cut through the domain are shown in Figure 6, where contours are separated by $2 \mathrm{~ms}$. As can be seen the shape of the wavefront far from the boundary is more affected by the diffusive inclusions than on the boundary of the domain.

Table 2: Various test cases and total depolarization time, $T_{D}$.

\begin{tabular}{|c|c|c|c|c|c|c|c|c|c|c|}
\hline & ref & \multicolumn{7}{|c|}{ Volume fraction from HR-MRI } & \multirow{2}{*}{\multicolumn{2}{|c|}{$\begin{array}{c}\text { Artificial scars } \\
\text { cubes }\end{array}$}} \\
\hline shapes & 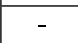 & $\begin{array}{l}\text { sticks }(\|) \\
\end{array}$ & plates $(\|)$ & plate & s $(\perp)$ & & cubes & & & \\
\hline$\sigma_{d}\left[S^{-1}\right.$ & - & \begin{tabular}{|l|l|}
0.02 & 0.3 \\
\end{tabular} & \begin{tabular}{|l|l|}
0.02 & 0.3 \\
\end{tabular} & 0.02 & 0.3 & 0.02 & 0.15 & 0.3 & 0.02 & 0.3 \\
\hline$T_{D}[\mathrm{~ms}]$ & 32.66 & \begin{tabular}{|l|l|}
33.02 & 31.75 \\
\end{tabular} & \begin{tabular}{l|l|}
32.26 & 30.99 \\
\end{tabular} & 44.89 & 44.07 & 34.31 & 32.49 & 33.3 & 34.91 & 34 \\
\hline
\end{tabular}
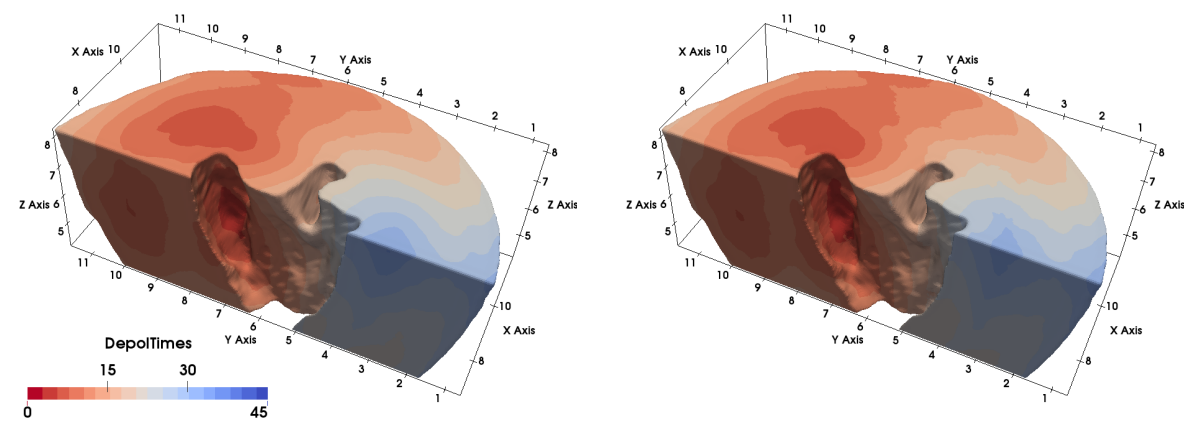

Fig. 5: Depolarization isochrones. From left to right, reference case and parallel plates, $\sigma_{d}=0.02 \mathrm{Sm}^{-1}$. Time between consecutive isochrones is $2.5 \mathrm{~ms}$. 

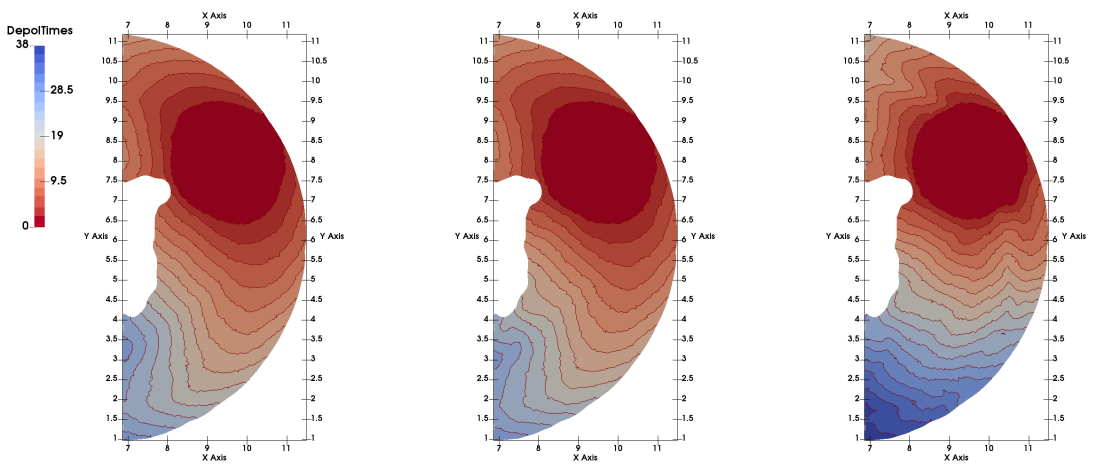

Fig. 6: Depolarization isochrones. From left to right: reference case, parallel plates and perpendicular plates, $\sigma_{d}=0.02 \mathrm{Sm}^{-1}$. Time between consecutive isochrones is $2 m s$.

\section{Conclusions and Discussion}

In some pathological cases the microscopic structure of the cardiac tissue is affected and there is an increase in collagen, fatty or fibrous tissue. These microscopic changes affect the propagation of electrical signals through the heart walls. In models, these changes are usually accounted for through the tuning of model parameters in an ad hoc way.

We have presented a modified bidomain model that has been derived in a rigorous way from the microscopic model of heterogeneous tissue. The modelling assumption is that the fibrous infiltrations are electrically passive, and are organized in a locally periodic way. Then, using the homogenisation technique the modified bidomain model has been derived, where the diffusive inclusions give rise to modified conductivity tensors in the bidomain model. We obtain a direct relation between the modified conductivity tensors and the local size and shape of the diffusive inclusions.

Further, we described a framework to obtain image based distribution of the parameters for the modified bidomain model. We worked on high resolution MRI of the rat heart. Using thresholding we detected the diffusive inclusions in the images and determined their local volume fractions. We used several test cases for the shapes of the inclusions, and computed the modified conductivity tensors for each test case, based on the local volume fractions of the diffusive inclusions. Finally, we ran simulations for all test cases and compared the results to the reference case, without diffusive inclusions.

The results are interesting as we could observe changes in the velocity of propagation, from $5 \%$ in some test cases to $37 \%$ in others. The largest impact on the velocity and shape of the depolarization wavefront occurred for diffusive inclusions perpendicular to the fiber directions. This is in agreement with previous $2 \mathrm{D}$ test cases in [6], where it has been observed that the principal direction of propagation might change depending on the shape and the large volume fraction 
of diffusive inclusions.

Since we do not have an a priori knowledge on the actual shapes of the diffusive inclusions, we have tested several simple geometries. To our knowledge, there has not been any studies performed that would give us a more precise idea on the shape of passive inclusions in the cardiac tissue.

In this paper we aimed to demonstrate the possibility to rigorously determine model parameters form HR-MRI of the rat heart with a pathological heterogeneous structure. It has a great deal of possible applications to fibrotic disease, ischemic heart disease, infarct scars etc.

Acknowledgments. The authors would like to thank Stephen Gilbert for providing HR-MRI and DT-MRI data of the rat heart, and for the useful discussions about the imaging process. This work was funded by the ANR project HR-CEM reference ANR-13-MONU-0004. This work was partially supported by an ANR grant part of "Investments d'Avenir" program reference ANR-10-IAHU-04.

\section{References}

1. Allaire G. Homogenization and two-scale convergence. SIAM Journal on Mathematical Analysis. 1992 Nov;23(6):1482-518.

2. Bensoussan A, Lions JL, Papanicolaou G. Asymptotic analysis for periodic structures. Amsterdam: North-Holland Publishing Company; 1978.

3. Patrizia Camelliti, Thomas K. Borg, and Peter Kohl. "Structural and functional characterisation of cardiac fibroblasts". Cardiovascular research 65.1 (2005), pp. 40-51.

4. Clayton RH, Bernus O, Cherry EM, Dierckx H, Fenton FH, Mirabella L, Panfilov AV, Sachse FB, Seemann G, Zhang H. Models of cardiac tissue electrophysiology: progress, challenges and open questions. Progress in biophysics and molecular biology. $2011 ; 104(1): 22-48$.

5. Coudiere Y, Davidovic A, Poignard C. The modified bidomain model with periodic diffusive inclusions. In Computing in Cardiology Conference (CinC), 2014 (pp. 10331036). IEEE.

6. Andjela Davidovic. Multiscale mathematical modelling of structural heterogeneities in cardiac electrophysiology. PhD Thesis, University of Bordeaux, 2016. https://hal. archives-ouvertes.fr/tel-01478145

7. Ethier M, Bourgault Y. Semi-implicit time-discretization schemes for the bidomain model. SIAM Journal on Numerical Analysis. 2008; 46(5):2443-68.

8. Gilbert SH, Benoist D, Benson AP, White E, Tanner SF, Holden AV, Dobrzynski $\mathrm{H}$, Bernus O, Radjenovic A. Visualization and quantification of whole rat heart laminar structure using high-spatial resolution contrast-enhanced MRI. American Journal of Physiology-Heart and Circulatory Physiology. 2012; 302(1):H287-98.

9. Mitchell CC, Schaeffer DG. A two-current model for the dynamics of cardiac membrane. Bulletin of mathematical biology. 2003; 65(5):767-93.

10. Keener JP, Sneyd J. Mathematical physiology. New York: Springer; 2009.

11. Neu JC, Krassowska W. Homogenization of syncytial tissues. Critical reviews in biomedical engineering. 1992; 21(2):137-99.

12. Ngoma DV, Bourgault Y, Nkounkou H. Parameter Identification for a Nondifferentiable Ionic Model Used in Cardiac Electrophysiology. Applied Mathematical Sciences. 2015; 9(150):7483-507. 
13. Pandit SV, Clark RB, Giles WR, Demir SS. A mathematical model of action potential heterogeneity in adult rat left ventricular myocytes. Biophysical journal. 2001; 81(6):3029-51.

14. Pennacchio M, Savare G, Franzone PC. Multiscale modeling for the bioelectric activity of the heart. SIAM Journal on Mathematical Analysis. 2005; 37(4):1333-70.

15. Rioux M, Bourgault Y. A predictive method allowing the use of a single ionic model in numerical cardiac electrophysiology. ESAIM: Mathematical Modelling and Numerical Analysis. 2013; 47(4):987-1016.

16. Relan J, Pop M, Delingette H, Wright GA, Ayache N, Sermesant M. Personalization of a cardiac electrophysiology model using optical mapping and MRI for prediction of changes with pacing. IEEE Transactions on Biomedical Engineering. 2011; 58(12):3339-49. 